\title{
Deuterium retention in tungsten films after different heat treatments
}

\author{
P. Wang ${ }^{1}$, W. Jacob ${ }^{2 *}$, S. Elgeti ${ }^{2}$ \\ ${ }^{1}$ State Key Laboratory of Solid Lubrication, Lanzhou Institute of Chemical Physics, Chinese Academy of \\ Sciences, Lanzhou, 730000, PR China \\ ${ }^{2}$ Max-Planck-Institut für Plasmaphysik, Boltzmannstr.2, 85748 Garching, Germany
}

\begin{abstract}
:
Tungsten films deposited by magnetron sputtering on polycrystalline tungsten substrates were used as a model system to study the influence of the film microstructure on deuterium retention behavior. Different microstructures were produced by annealing the films up to recrystallization temperature and the corresponding structural changes were investigated by scanning electron microscopy combined with focused ion beam (FIB) cross sectioning. The influence of the induced structural changes on D retention was investigated by both nuclear reaction analysis and temperature-programmed desorption. $\mathrm{D}$ concentration in the investigated $\mathrm{W}$ films is higher than in polycrystalline bulk tungsten by a factor of 3 . D retention in the films decreases as a function of annealing temperature. After annealing at $2000 \mathrm{~K}$, FIB cross-section images reveal that cavities appeared at the grain boundaries within the film and at the initial interface between the $\mathrm{W}$ film and $\mathrm{W}$ substrate. This new microstructure strongly affects the $\mathrm{D}$ depth profile and leads to the increase of $\mathrm{D}$ retention. Although a further increase of the holding time at $2000 \mathrm{~K}$ or an increase of the annealing temperature to $2150 \mathrm{~K}$ lead to the reduction of the retained $\mathrm{D}$ amount, the $\mathrm{D}$ concentration in the recrystallized $\mathrm{W}$ films cannot be reduced to a level as low as that of bulk W recrystallized at $2000 \mathrm{~K}$ for $30 \mathrm{~min}$.
\end{abstract}

PACS: 81.15.Cd, 52.40.Hf, 28.52.Fa, 79.20.Rf

Keywords: Tungsten; Films, Microstructure, Annealing, Deuterium retention

Published in: $\quad$ Journal of Nuclear Materials, 456, 192-199 (2014).

doi: $\quad$ http://dx.doi.org/10.1016/j.jnucmat.2014.09.023

$\begin{array}{ll}\text { Submitted: } & 07.04 .2014 \\ \text { Accepted: } & 11.09 .2014 \\ \text { Available online: } & 19.09 .2014\end{array}$

\footnotetext{
${ }^{*}$ Corresponding author. Fax:+49-89-32991504, Tel:+49-89-32992618.

E-mail address: Wolfgang.Jacob@ipp.mpg.de
} 


\section{Introduction}

Retention of large quantities of tritium in plasma-facing components of future thermonuclear fusion devices increases the fuel costs and presents a safety concern. So understanding the fuel retention properties of plasma-facing materials is important for ITER and next-step fusion devices such as DEMO [1-3]. Due to its favorable physical properties, such as low erosion yield and high melting temperature, tungsten will be used as plasmafacing material in the ITER divertor and it is considered as the most promising plasma-facing material in future fusion devices [1,2]. Because the solubility of hydrogen isotopes in tungsten is very low trapping at defects determines the retention [3-6]. These defects are either natural defects which are present after fabrication or they may be created by treatment after fabrication such as for example radiation-induced defects [3-6]. Many aspects of hydrogen retention in tungsten $(\mathrm{W})$ have been investigated in the past and the available literature is extensive (see reference in the review articles [3-6]).

In our previous work, $\mathrm{W}$ films deposited by magnetron sputtering were used to study the deuterium retention behavior and to compare with bulk $\mathrm{W}[7,8]$. There are several reasons to investigate $\mathrm{H}$ retention in $\mathrm{W}$ films. Firstly, up to now, tungsten coatings were used as the firstwall material in ASDEX Upgrade and JET. Secondly, in future fusion devices W will inevitably be sputtered and re-deposited on nearby surfaces and we can assume that the structure of re-deposited $\mathrm{W}$ layers in fusion devices is similar to $\mathrm{W}$ films produced by magnetron sputtering. And finally, $\mathrm{W}$ films allow the investigation of thin $\mathrm{W}$ layers which are thinner than layers that can be produced from bulk material. For some investigation of basic physical processes such model systems are advantageous [7].

Apart from the radiation-induced damage created during operation due to the intense neutron irradiation with high energy neutrons from the fusion reaction, hydrogen isotopes retention in $\mathrm{W}$ materials depends on the microstructure which is largely determined by the production history and the microstructural modifications during operation. For example, Manhard et al. have shown that a modification of the initial microstructure of their investigated tungsten grade has a big influence on the measured D retention [9]. Since firstwall materials in a future fusion device will be subjected to intense fluxes of incident particles and thermal loading [1,2], structural changes have to be anticipated. Consequently, the effect of such changes in the microstructure on fuel retention in $\mathrm{W}$ materials should be taken into account. The here investigated sputter-deposited $\mathrm{W}$ films can be considered as a very defectrich material and the microstructure of these films can be modified by annealing.

Accordingly, in this work, thin magnetron-sputter-deposited tungsten films were used as a model system for studying some aspects of the influence of changes of the film microstructure on the deuterium retention behavior. W films with different microstructure were produced by different heat treatments after deposition. The films were in the following loaded with D applying a well-characterized deuterium plasma. After D implantation both nuclear reaction analysis and temperature-programmed desorption were used to detect the $\mathrm{D}$ retention depth profile and total retained D amount.

\section{Experimental details}

Tungsten films with thicknesses of about $2.9 \pm 0.1 \mu \mathrm{m}$ were deposited using a commercial sputtering device (Discovery ${ }^{\circledR} 18$, Denton). The system was pumped down to a base pressure of less than $3 \times 10^{-5} \mathrm{~Pa}$ and the deposition was performed in argon atmosphere at $5 \times 10^{-1} \mathrm{~Pa}$. A liquid $\mathrm{N}_{2}$ cold trap was attached to the deposition chamber to reduce the water partial pressure. Prior to deposition the substrates were etched by an argon plasma at $-600 \mathrm{~V}$ bias for 2 minutes to remove the native oxide layer. During W deposition the dc power applied to the tungsten sputter target was kept constant at $300 \mathrm{~W}$. At these settings the deposition rate of $\mathrm{W}$ is about $17 \mathrm{~nm} / \mathrm{min}$. Sample rotation during deposition was used to produce layers with a homogenous 
thickness distribution. Neither extra substrate bias nor heating or cooling were applied during deposition. In this work, rolled $0.8 \mathrm{~mm}$ thick polycrystalline tungsten samples (base material manufactured by PLANSEE Metall GmbH, 6600 Reutte, Austria) with a guaranteed purity of 99.97 wt.\% were used as substrates. The samples were chemo-mechanically polished to a mirror-like finish and cleaned in an ultrasonic bath with acetone [10]. Prior to $\mathrm{W}$ film deposition the samples were stress-relieved and degassed by heating for 2 hours at $1200 \mathrm{~K}$ in a vacuum oven $\left(\mathrm{p}<5 \times 10^{-5} \mathrm{~Pa}\right)$. More details about the preparation of reference samples can be found in Ref. [10]. For comparison, D retention in an uncoated reference sample that has received the identical pre-treatment as the $\mathrm{W}$ substrates and in a recrystallized $\mathrm{W}$ sample annealed for $30 \mathrm{~min}$ at $2000 \mathrm{~K}$ were also investigated.

To change the initial structure of as-deposited tungsten films, different annealing treatments were carried out in two different vacuum ovens. One is a commercial graphite oven with protective gas atmosphere manufactured by Thermal Technology Company. The sample was wrapped by an envelope made of tungsten foil and then enclosed in a tantalum box to avoid carbon contamination. During the heating process argon was introduced as a protective gas. The maximum achievable temperature in this oven was $2000 \mathrm{~K}$. The second oven is a vacuum oven with a base pressure of about $2 \times 10^{-6} \mathrm{~Pa}$. At the maximum operation temperature of about $2150 \mathrm{~K}$ the background pressure raises to about $2 \times 10^{-5} \mathrm{~Pa}$. The temperature of the sample can be increased from room temperature to $2150 \mathrm{~K}$ using electron beam heating. Sample temperatures are measured by disappearing filament pyrometer focused onto the edge of the sample.

Scanning electron microscopy (SEM) was used to investigate the structure of as-deposited and annealed films. The used SEM (Helios NanoLab 600, FEI) allows in situ cross-sectioning of samples by the implemented focused ion beam (FIB). The secondary electrons produced by a $5 \mathrm{keV}$ electron beam were detected by the inlens detector system. The cross sections were imaged with the e-beam tilted by $38^{\circ}$ to their surface plane, so that the vertical scale in the shown images is compressed relative to the horizontal scale.

Deuterium implantation was carried out in the laboratory plasma experiment PlaQ. A basic description of $\mathrm{PlaQ}$ is given in [11-13]. The detailed experimental procedures applied for samples investigated in this article are described in [7]. In short, PlaQ consists of a stainless steel chamber and is equipped with a remote electron cyclotron resonance (ECR) plasma source. A diverging plasma beam impinges perpendicularly onto the substrates which are located $100 \mathrm{~mm}$ below the cage exit. The energy of the ions impinging on the substrates was controlled applying a dc bias. Samples are tightly clamped to the holder by four molybdenum screws to optimize thermal contact. Sample temperatures are measured by a thermocouple attached to the sample holder from the back side and by an infrared camera viewing the sample surface. The temperature measured by the IR camera is calibrated against the thermocouple temperature in steady state without plasma operation. At the used $\mathrm{D}_{2}$ gas pressure the deuteron flux consists dominantly of $\mathrm{D}_{3}{ }^{+}$ions $(94 \%$ of the impinging ions corresponding to $97 \%$ of the impinging deuterons in form of ions) with minor contributions of $\mathrm{D}_{2}^{+}$(2\% of the deuterons) and $\mathrm{D}^{+}$(1\% of the deuterons) [13]. In this article we refer to the energy per deuterium atom of the dominant molecular ion species as the ion energy. For the implantation performed at a dc substrate bias of $-100 \mathrm{~V}$ this results, together with the plasma potential of about $-15 \mathrm{~V}$ [13], in an ion energy of about $115 \mathrm{eV}$. This corresponds to a mean energy of about $38 \mathrm{eV}$ per deuteron for the dominant $\mathrm{D}_{3}{ }^{+}$ions. The deuteron flux for these settings is $9 \times 10^{19} \mathrm{Dm}^{-2} \mathrm{~s}^{-1}$ [13]. In the experiments described here all deuterium implantations were carried out at a sample temperature of $370 \mathrm{~K}$

To measure the density of the investigated magnetron-sputtered W films some films were deposited simultaneously on both silicon and tungsten substrates. Rutherford backscattering spectrometry (RBS) was applied to measure the atomic areal density of the film deposited on the silicon substrate. A beam of $4.0 \mathrm{MeV}^{4} \mathrm{He}$ was used at normal incidence and a scattering 
angle of $165^{\circ}$. A charge of $15 \mu \mathrm{C}$ was usually accumulated for one RBS spectrum to reach reasonable counting statistics. The total atomic areal density of the $1.18 \mu \mathrm{m}$ thick $\mathrm{W}$ film determined by RBS is $(6.7 \pm 0.3) \times 10^{22}$ atoms $/ \mathrm{m}^{2}$. From the thickness and the areal density we calculate a mass density of $(17.4 \pm 0.9) \mathrm{g} / \mathrm{cm}^{3}$. For the $\mathrm{W}$ film deposited on W substrate, a mass density of $(17.6 \pm 0.5) \mathrm{g} / \mathrm{cm}^{3}$ was derived based on the mass change due to deposition and the film thickness from stylus profilometry. This value is in good agreement with the value determined from RBS. Both measurements show that the density of our $\mathrm{W}$ films is about $10 \%$ lower than that of bulk W $\left(19.3 \mathrm{~g} / \mathrm{cm}^{3}\right)$.

The depth distribution of the retained deuterium was measured by NRA by means of the $\mathrm{D}\left({ }^{3} \mathrm{He}, \alpha\right) \mathrm{p}$ nuclear reaction two months after implantation. The $\mathrm{D}$ concentration within the near-surface layer (for depths up to about $0.3 \mu \mathrm{m}$ ) was determined at a ${ }^{3} \mathrm{He}$ energy of $0.69 \mathrm{MeV}$ by analyzing the emitted $\alpha$ particles with a surface barrier detector at the laboratory scattering angle of $102^{\circ}$ equipped with a rectangular slit reducing the solid angle to $9.16 \mathrm{msr}$. For determining the $\mathrm{D}$ concentration at larger depths, the energy of the analyzing beam of ${ }^{3} \mathrm{He}$ ions was varied from 0.69 to $4.5 \mathrm{MeV}$. In this work, the following ion beam energies were used in the analysis of the thickest W films: $0.69,1.2,1.8,2.4,3.2,4.5 \mathrm{MeV}$. A charge of $10 \mu \mathrm{C}$ was usually accumulated for one NRA spectrum. The high energy protons from the $\mathrm{D}\left({ }^{3} \mathrm{He}, \mathrm{p}\right){ }^{4} \mathrm{He}$ nuclear reaction were counted using a thick, large angle solid state detector at a scattering angle of $135^{\circ}$ equipped with a curved slit reducing the solid angle to $29.9 \mathrm{msr}$. In order to analyze the D concentration profile in the whole layer both SIMNRA [14] and NRADC [15] were used for the deconvolution of the NRA spectra measured at different ${ }^{3} \mathrm{He}$ ion energies. As input data for NRADC we use the $\alpha$ spectrum measured at $0.69 \mathrm{MeV}$ and all proton spectra measured at the different energies. Details about the data evaluation using NRADC can be found in Ref. [7]. The total amount of D retention was finally determined by integrating the D profile over the measured depth.

After the NRA measurement the retained D amount samples was additionally measured by temperature programmed desorption (TPD) in the quartz tube of the TESS device. A basic description of TESS is given in [16]. Details of the experimental procedures applied for the results presented in this article can be found in Ref. [7]. The samples were heated up to a sample temperature of $1275 \mathrm{~K}$ with an oven heating rate of $15 \mathrm{~K} / \mathrm{min}$. The desorbed gases were measured with a quadrupole mass spectrometer (QMS). Selected mass channels between 2 and $44 \mathrm{amu}$ were recorded as a function of time. For the quantitative analysis the QMS signals for $\mathrm{HD}, \mathrm{D}_{2}$ and $\mathrm{CD}_{4}$ were calibrated as described in [7]. The calibration factors for $\mathrm{HD}$ and $\mathrm{D}_{2}$ are $(3.5 \pm 0.14) \times 10^{9}$ molecules per count, and $(3.8 \pm 0.15) \times 10^{9}$ molecules per count, respectively. We assume that the QMS calibration for $\mathrm{HD}, \mathrm{H}_{2}$ and $\mathrm{D}_{2}$ has an uncertainty of less than $10 \%$.

\section{Results and discussion}

\subsection{Microstructures of W films}

To avoid possible structural variations due to the different deposition runs all $\mathrm{W}$ films used in this work were deposited in one single batch on hot-rolled polycrystalline tungsten substrates. After deposition the samples were annealed to different temperatures. The microstructures of $\mathrm{W}$ films before and after annealing were investigated by FIB cross sectioning. Fig. 1 shows cross-section images of $\mathrm{W}$ films deposited on bulk $\mathrm{W}$ before and after different heat treatments. For the as-deposited W film two different features can be distinguished from the cross-section image (Fig. 1a). The lower part of image shows the bulk $\mathrm{W}$ substrate, in which the grain boundaries are oriented predominantly parallel to the sample surface. There, all grains show an elongation in the rolling direction. While the average length of the grains is about 0.5 to $5 \mu \mathrm{m}$ the mean thickness is only about $0.4 \mu \mathrm{m}$. At the interface between $\mathrm{W}$ film and substrate, indicated by the short dashed line on the left side of the figure, 
three elongated grains are discernible. One of these grains is outlined by a dashed line in Fig. 1a. These grains belong to the $\mathrm{W}$ substrate and show a somewhat irregularly shaped bottom as other grains in the $\mathrm{W}$ bulk and a flat upper surface which is produced by mechanical polishing during substrate pre-treatment. Above this flat interface the about $2.9 \mu \mathrm{m}$ thick $\mathrm{W}$ film is visible. Obviously, the growth of the $\mathrm{W}$ film is strong affected by the grains of the underlying $\mathrm{W}$ substrate and a typical epitaxial growth of the deposited $\mathrm{W}$ film on the substrate grains is found. This is expected because the film and the bulk material are identical so that the lattice parameters match. To facilitate epitaxial growth the interface has to be well prepared, such that no distorted layer from polishing is present, and that the substrate surface has to be sufficiently clean, such that, e.g. no oxide layer is present prior to deposition. The observation of epitaxial growth in our samples may be taken as confirmation that our sample preparation procedure produces very good surfaces. Initially, each growing W grain has similar lateral dimension as the corresponding underlying grain. During growth different grains have different growth rates in different crystal orientations resulting in a change of the lateral dimension and a slight roughening of the surface. In some cases, new grains nucleate within the growing film. Within the $\mathrm{W}$ film the grain boundaries are oriented preferably in the direction normal to the substrate surface which can be explained by columnar growth.
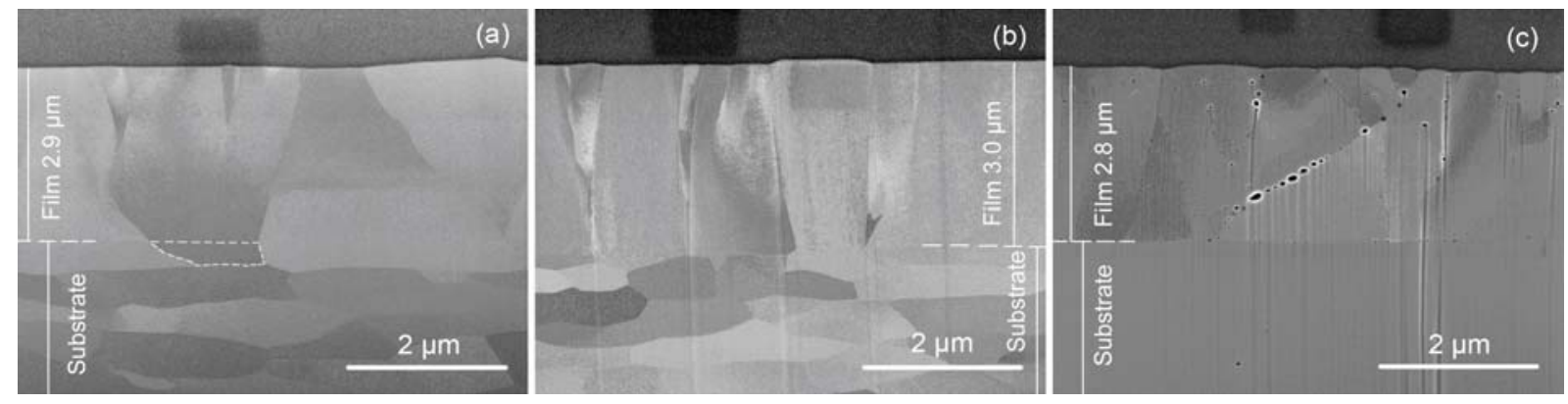

Fig. 1: Cross-section images of $W$ film (upper part of the image) deposited on bulk $W$ (lower part): (a) as-deposited, (b) annealed at $1200 \mathrm{~K}$ for $30 \mathrm{~min}$ and (c) $2000 \mathrm{~K}$ for $30 \mathrm{~min}$. The interface between the film and substrate is indicated by the short horizontal line. The film thickness of $2.9 \pm 0.1 \mu \mathrm{m}$ is indicated by the vertical line. Note that the angle between the electron beam and the cross-section plane is $38^{\circ}$ so that the vertical distances appear in the images shorter that they are in reality.

The cross-section image of the $\mathrm{W}$ film after $1200 \mathrm{~K}$ annealing is shown in Fig. $1 \mathrm{~b}$, no obvious changes are visible within the $\mathrm{W}$ bulk substrate. This is not surprising because, on the one hand, it was shown by Manhard $[9,10]$ that the grain structure of this $\mathrm{W}$ grade does not change due to annealing at $1200 \mathrm{~K}$ and, on the other hand, the bulk sample was already annealed for 2 hours at $1200 \mathrm{~K}$ during substrate preparation. The initial interface between the $\mathrm{W}$ film and the $\mathrm{W}$ bulk is hardly visible in Fig. $1 \mathrm{~b}$ but it is still discernible in the original image on the computer screen. This is due to the fact that the contrast between the top grain of the substrate and the film has almost vanished. What remains clearly discernible are the lower grain boundaries of the top grains of the substrate. This leaves us with the wrong impression of a somewhat rough interface. The grain structure within the film is not significantly different from the as deposited film. Still, grain boundaries are oriented preferably perpendicular to the interface.

For the $\mathrm{W}$ film annealed at $2000 \mathrm{~K}$ for 30 minutes, the cross-section image is quite unexpected. Typically, for pure tungsten recrystallization sets in for temperatures higher than $1700 \mathrm{~K}$ [9]. Due to recrystallization the density of both dislocations and grain boundaries decreases and the grains grow. Manhard et al. have investigated the microstructure of the here used polycrystalline $\mathrm{W}$ material and the influence of annealing $[9,10]$. Grains in the nonrecrystallized material were small with typical dimensions ranging from less than $1 \mu \mathrm{m}$ up to 
several $\mu \mathrm{m}$. Typical grain dimensions after recrystallization at $2000 \mathrm{~K}$ are between 10 and $50 \mu \mathrm{m}$. This is in agreement with the interpretation of the lower part of Fig. 1c which belongs to the $\mathrm{W}$ substrate. The grain shown in the lower part of Fig. 1c which has formed during recrystallization is much larger than the shown image size. But contrary to expectation the sputter-deposited $\mathrm{W}$ film is still clearly discernible from the bulk. Even after annealing at $2000 \mathrm{~K}$ many relatively small grains exist in the film and the interface to the bulk is clearly visible. The grain size distribution is not dramatically different from the as-deposited and $1200 \mathrm{~K}$ annealed film. Surprisingly, new cavity structures with diameters ranging from ten to hundred nm appear and these cavities accumulate at the grain boundaries within the $\mathrm{W}$ films and at the interface between the $\mathrm{W}$ film and substrate.

Two processes could contribute to the observed cavity formation after high temperature annealing. The first one is the accumulation of gaseous impurities and the second one is the movement of volume defects such as vacancies, voids and dislocations. Since the impurity concentration (mainly oxygen, argon and carbon) in the deposited films is below $1 \%$ (see Ref. [7]), it is hard to believe that the cavities are formed due to the accumulation of impurities. The second possible explanation is that these cavities could result from the movement of defects at very high temperature. As mentioned above, the density of our W coatings produced by magnetron sputtering is $10 \%$ lower than the density of bulk W. This suggests that many defects are present within the as-deposited film. These defects can be vacancies, dislocations, vacancy clusters, or more extended 3-dimensional voids which are, however, too small to be visible in our SEM images. During recrystallization these defects could become mobile and form new 3-dimensional structures (cavities) which are so large that they are visible in the SEM images. Obviously these newly formed cavities preferably accumulate at the grain boundaries and the interface. These results presented in Fig. 1c reveal that the recrystallization process for our $\mathrm{W}$ coating is incomplete or inefficient at $2000 \mathrm{~K}$, and that this sputter-deposited W film shows a higher resilience against recrystallization than our hotrolled polycrystalline $\mathrm{W}$ which is used as substrate material.

\subsection{Deuterium retention}

Five different samples were simultaneously exposed to deuterium plasma at $370 \mathrm{~K}$ substrate temperature with $38 \mathrm{eV} / \mathrm{D}$ incident energy and a fluence of $6 \times 10^{24} \mathrm{D} / \mathrm{m}^{2}$. The samples were three W films on bulk W substrates (as-deposited, annealed at $1200 \mathrm{~K}$ and annealed at $2000 \mathrm{~K}$ ), an uncoated substrate that received the identical pre-treatment as the substrates (see section 2), and an uncoated substrate additionally annealed at $2000 \mathrm{~K}$ for 30 min. After implantation, deuterium retention in these $\mathrm{W}$ samples with different structures was measured by both NRA and TPD. The deuterium retention measurements were performed two months after implantation. That means all detected deuterium atoms should be retained in deep traps $[5,6,17]$.

Fig. 2 shows the D concentration depth profiles determined by NRA. On top of all samples we find a D-rich surface layer with an areal density of $1 \times 10^{21} \mathrm{D} / \mathrm{m}^{2}$. The thickness of this top layer cannot be resolved as it is thinner than the depth resolution of the $\alpha$ spectrum measured at $0.69 \mathrm{MeV}$. More details can be found in Ref. [7]. Below this D-rich surface layer the $\mathrm{D}$ depth profiles show that the local $\mathrm{D}$ concentrations in the different investigated $\mathrm{W}$ materials vary by more than two orders of magnitude from $10^{-5}$ to $10^{-2}$ (atomic fraction). The two reference samples, stress-relieved hot-rolled polycrystalline tungsten and recrystallized bulk tungsten $(2000 \mathrm{~K}, 30 \mathrm{~min})$ both show much lower D concentration than the comparable coated samples. Deuterium retention in the stress-relieved polycrystalline $\mathrm{W}$ is inhomogeneous in depth. In the depth range up to about $4 \mu \mathrm{m}$ the $\mathrm{D}$ concentration in bulk W is about $1-2 \times 10^{-3}$ and for larger depths the concentration drops by about one order of magnitude to $10^{-4}$. Recrystallization reduces the $\mathrm{D}$ concentration in bulk W to less than $10^{-4}$ in the top $4 \mu \mathrm{m}$ and to about $2-3 \times 10^{-5}$ for larger depth. It should be mentioned that the latter 
value already approaches the detection limit of the NRA method for these types of samples. We conclude that most of the defects which are able to trap D in the recrystallized bulk W were annealed after $30 \mathrm{~min}$ at $2000 \mathrm{~K}$. This conclusion is in excellent agreement with the observations of Manhard et al. [9].

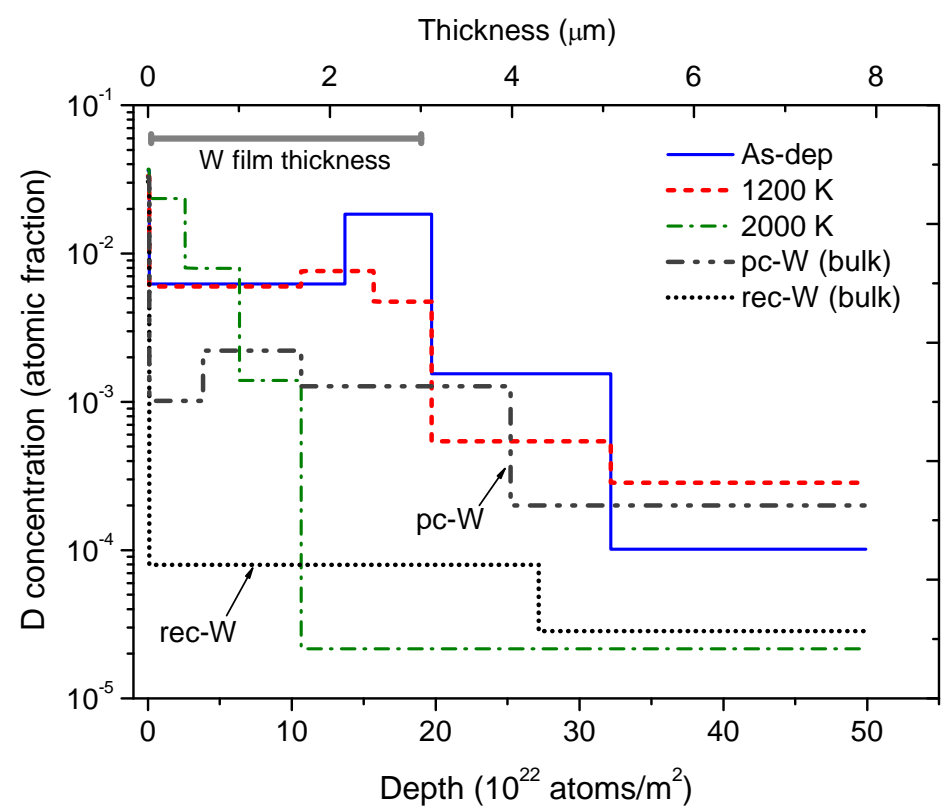

Fig. 2: Deuterium concentration depth profiles of $W$ coatings before and after annealing treatments. The D depth profiles of two bulk $W$ samples annealed at $1200 \mathrm{~K}$ and $2000 \mathrm{~K}$ are also shown for reference. All implantations were performed at $370 \mathrm{~K}$ implantation temperature with $38 \mathrm{eV} / \mathrm{D}$ incident energy with a fluence of $6 \times 10^{24} \mathrm{D} / \mathrm{m}^{2}$.

The D concentration in the as-deposited $\mathrm{W}$ film is below the common D-rich near surface layer up to a depth of about $2 \mu \mathrm{m}$ relatively constant at $6 \times 10^{-3}$. This concentration is by about a factor of 3 higher than the D concentration in the reference $\mathrm{W}$ sample and almost 2 orders of magnitude higher than in recrystallized $\mathrm{W}$ bulk material. Close to the interface the D concentration increases to $2 \times 10^{-2}$. This enhancement could be due to the formation of blisters in the vicinity of the interface. For this sample many blisters were found on SEM surface images. In a recent publication [8] it was shown that these blisters are located close to the interface inside the $\mathrm{W}$ bulk material and not in the film or at the interface. These blisters lead to an increase of the D concentration in the vicinity of the interface.

The higher $\mathrm{D}$ retention in the $\mathrm{W}$ film is attributed to a higher defect density in the films as compared with the $\mathrm{W}$ bulk material. The $\mathrm{W}$ film annealed at $1200 \mathrm{~K}$ shows a similar D profile as the as-deposited $\mathrm{W}$ film. Both samples exhibit a relatively constant $\mathrm{D}$ depth distribution within the film. A notable difference is that the D-accumulated zone in the region of the interface of the as-deposited $\mathrm{W}$ film is absent in the $\mathrm{W}$ film after $1200 \mathrm{~K}$ annealing. In the corresponding surface images of this layer no blisters were found after D implantation whereas blisters were found in the as-deposited samples. That means in the $1200 \mathrm{~K}$ annealed $\mathrm{W}$ film blister formation at interface is suppressed and simultaneously the increase of the D concentration in the region of the interface is not observed. This observation is in agreement with the interpretation that the increased $\mathrm{D}$ concentration at the interface is related to the appearance of blisters. The difference between the depth profiles for these two samples for depths larger than $3 \mu \mathrm{m}$ is within the uncertainty of the deconvolution procedure. In that respect it has to be kept in mind that for these two samples more than $80 \%$ of the total retained $\mathrm{D}$ amount is within the film (see discussion below). 


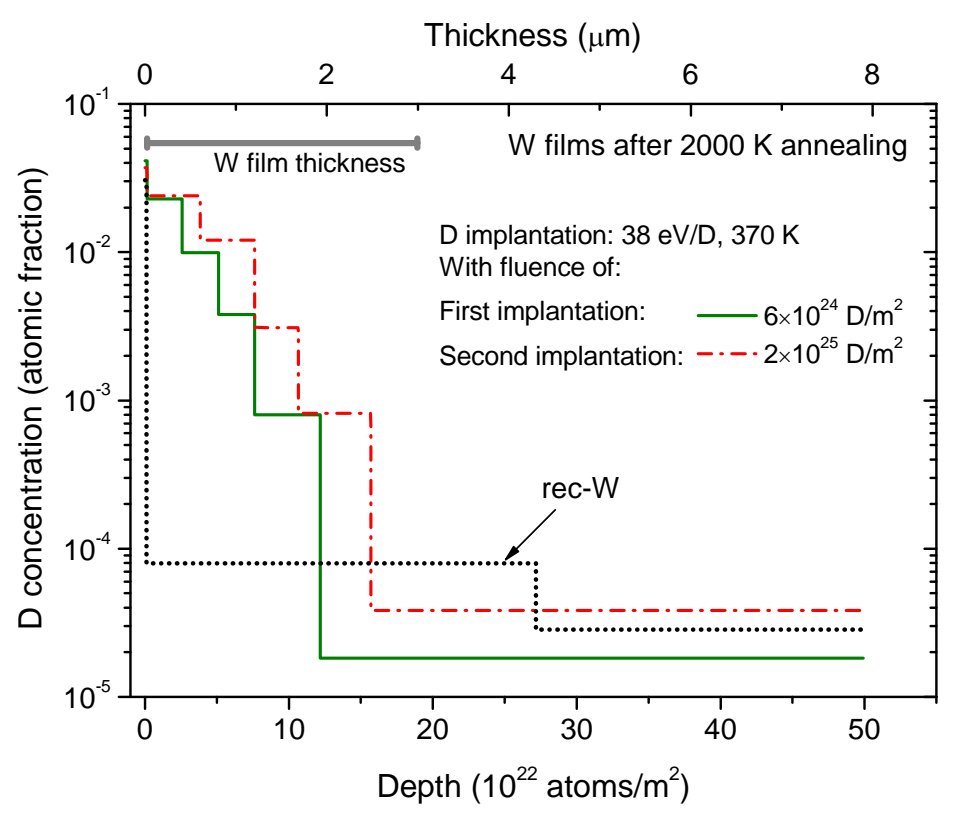

Fig. 3: Deuterium concentration depth profiles of recrystallized $W$ films implanted at $370 \mathrm{~K}$ with $38 \mathrm{eV} / \mathrm{D}$ incident energy with fluence of $6 \times 10^{24}$ and $2 \times 10^{25} \mathrm{D} / \mathrm{m}^{2}$, respectively. The second implantation was performed on the identical sample after TPD measurement. The D depth profile of bulk $W$ annealed at $2000 \mathrm{~K}$ and implanted to a fluence of $6 \times 10^{24} \mathrm{D} / \mathrm{m}^{2}$ is shown for reference.

The $\mathrm{D}$ depth profile of the $\mathrm{W}$ film annealed at $2000 \mathrm{~K}$ is distinctly different from that of the other two $\mathrm{W}$ films. Close to the surface in the region below the very thin D-rich surface layer up to about a depth of $1 \mu \mathrm{m}$ the D concentration is even higher than in the other two W film samples. In the first $400 \mathrm{~nm}$ the $\mathrm{D}$ concentration is $2.3 \times 10^{-2}$ which is a factor of 4 higher than in the as-deposited and $1200 \mathrm{~K}$ annealed films. With increasing depth the D concentration decreases strongly. For depth larger than $1.8 \mu \mathrm{m}$ the $\mathrm{D}$ concentration drops to values comparable to those of the recrystallized bulk $\mathrm{W}$ samples without $\mathrm{W}$ film. Considering the fact that a lot of cavities were observed in this sample (Fig. 1c), and both surface and cross-section images prove that no blisters were formed after D implantation, the observed high $\mathrm{D}$ concentration is obviously due to the presence of the cavities. Taking further into account that after annealing at $2000 \mathrm{~K}$ most lattice defects which contribute to D retention should have vanished [9] such that this enhanced D retention cannot be explained to D in the bulk surrounding the cavities, it can only be attributed to $\mathrm{D}$ retention inside these large cavities visible by SEM (Fig. 1c). Based on the available data we cannot distinguish whether $\mathrm{D}$ is dominantly adsorbed on the inner surfaces of the cavities or as molecules inside the cavity volume.

In Fig. 1c we can see that the cavities occur in all depths of the film up to the interface. If we assume that the depth distribution of the cavity volume within the $\mathrm{W}$ film is homogeneous, then we should measure a flat deuterium depth profile within the film if all cavities are equally filled. But the D depth profile for the film annealed at $2000 \mathrm{~K}$ (Fig. 2) shows a steep decay within the top $2 \mu \mathrm{m}$. So either the cavities are not yet fully filled, or the assumption of a homogeneous depth distribution of the cavities is wrong. To check these two hypotheses the identical sample was reloaded with D after TPD outgassing. Since during TPD the maximum sample temperature is $1275 \mathrm{~K}$ we assume that the microstructure of this sample, which was previously annealed at $2000 \mathrm{~K}$, was not changed during TPD. In this second loading experiment the sample was exposed to an about 3 times higher fluence $\left(2 \times 10^{25} \mathrm{D} / \mathrm{m}^{2}\right)$ compared with the first loading. This D implantation took 63 hours. The D concentration 
depth profiles of this sample after the first and the second loading cycle are plotted in Fig. 3 . Obviously, after the longer D implantation, i.e., the larger D fluence, D has penetrated deeper. The retained $\mathrm{D}$ amount measured by NRA after implantation with the 3-times higher fluence is about $50 \%$ higher. These results show that the first exposure at 3-times lower fluence was not sufficient to fill all possible traps for D. In this context it should be emphasized that a fluence of $6 \times 10^{24} \mathrm{D} / \mathrm{m}^{2}$ is sufficient to fill the as-deposited and $1200 \mathrm{~K}$-annealed sputterdeposited $\mathrm{W}$ layer up to the full layer thickness. Although this observation is not a prove that the trap profile within the $2000 \mathrm{~K}$-annealed W layer is homogeneous, it demonstrates that a further filling of the trap profile at larger fluence is possible. We conclude from this observation that the cavities are still not completely filled. It appears that the presence of these large cavities significantly slows down the penetration of D into this layer.

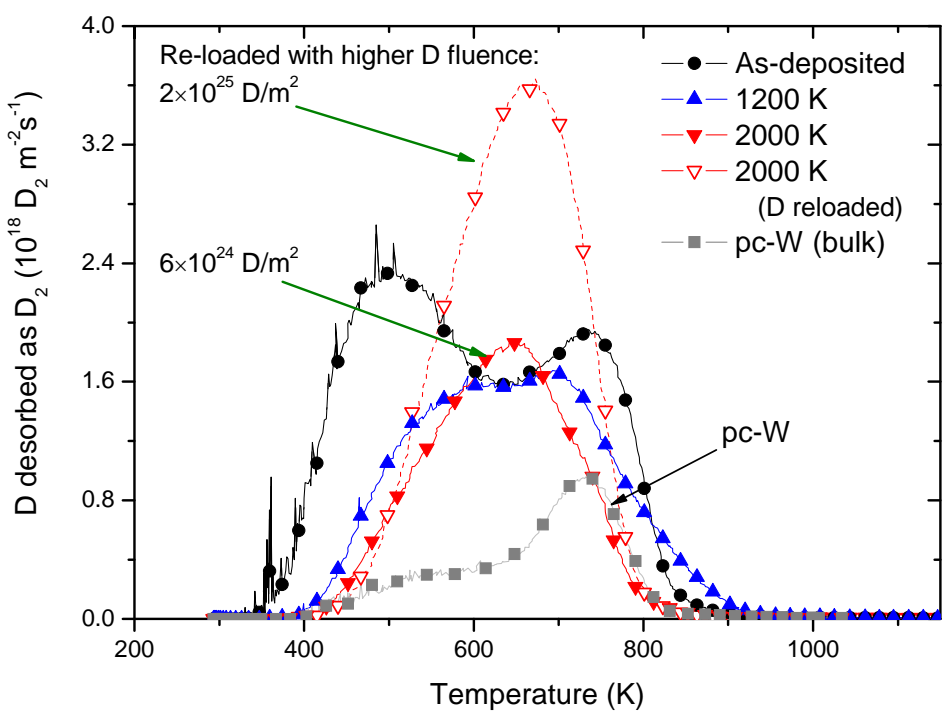

Fig. 4: Deuterium release spectra of $W$ coatings before and after annealing treatments. For comparison the $D_{2}$ release spectra of $p c-W$ is also shown. The second implantation to the fluence of $2 \times 10^{25} \mathrm{D} / \mathrm{m}^{2}$ for the sample annealed at $2000 \mathrm{~K}$ was performed on the identical sample after TPD measurement. In all cases, the oven heating rate is $15 \mathrm{~K} / \mathrm{min}$.

After NRA measurement the total retained D amount was determined by temperatureprogrammed desorption. Fig. 4 shows the $\mathrm{D}_{2}$ release spectra as a function of sample temperature. The reference polycrystalline bulk $\mathrm{W}$ shows the main $\mathrm{D}_{2}$ release peak at $730 \mathrm{~K}$ and a shoulder peak at around $550 \mathrm{~K}$. The as-deposited $\mathrm{W}$ film also shows two $\mathrm{D}_{2}$ release peaks at 500 and $730 \mathrm{~K}$, but the intensities of these two peaks are higher than those in bulk W. Additionally, a lot of sharp release peaks (spikes) appear at the low temperature part of this TPD spectrum. Such spikes, although at a much lower rate and intensity, are also discernible on the TPD spectrum of the reference sample. It has been shown earlier that such spikes occurring in this temperature range are due to the bursts of gas-filled blisters [18]. The appearance of two desorption peaks suggests the presence of at least two trap energies. They are typically assigned to deuterium trapped in intrinsic defects such as grain boundaries and dislocations [5]. For the $\mathrm{W}$ film annealed at $1200 \mathrm{~K}$, the intensity of both $\mathrm{D}_{2}$ release peaks decreases and the position of both release peaks shifts to 550 and $700 \mathrm{~K}$, respectively. We assume that the reduction of the peak intensity is due to the suppression of blister formation at the interface. This interpretation is in agreement with the interpretation of the $\mathrm{D}$ depth profile in Fig. 2 and the disappearance of spikes in the low temperature range due to the burst of blisters. For the $\mathrm{W}$ film after $2000 \mathrm{~K}$ annealing, only one broad $\mathrm{D}_{2}$ release peak is found at 
about $650 \mathrm{~K}$. Although the maximum is higher, the peak integral, i.e., the total released D amount, is about $25 \%$ lower than that of the $1200 \mathrm{~K}$ film. The total D amount in the $2000 \mathrm{~K}$ annealed W film is about twice the amount in the not recrystallized substrate. According to TPD the released D amount measured after implantation with the 3-times higher fluence in the $2000 \mathrm{~K}$ annealed sample has increased from $1.0 \times 10^{21} \mathrm{D} / \mathrm{m}^{2}$ to $1.8 \times 10^{21} \mathrm{D} / \mathrm{m}^{2}$.

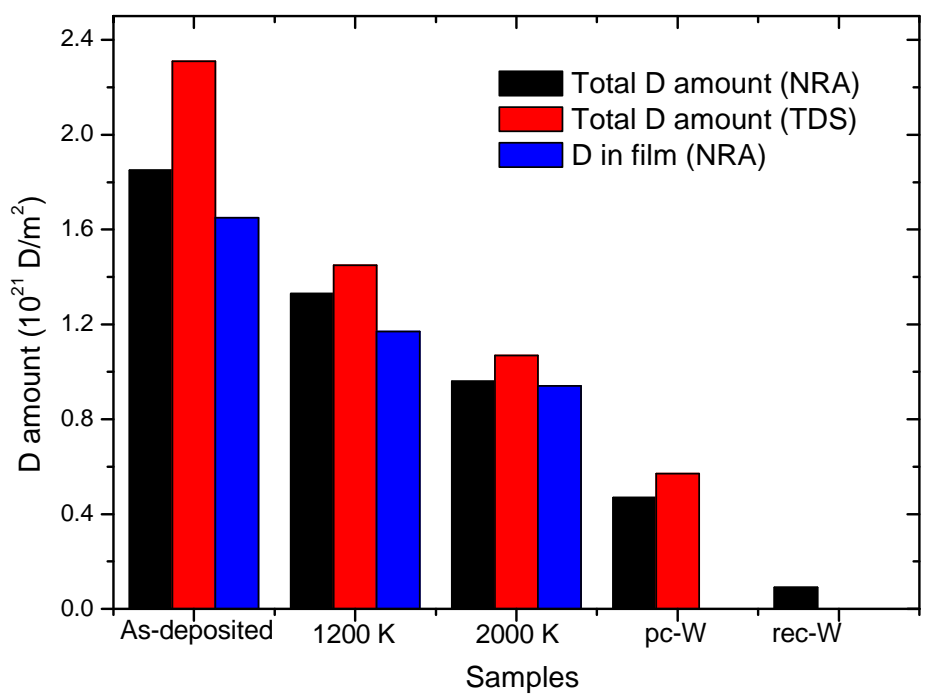

Fig. 5: Total retained deuterium amounts measured by both NRA and TPD. The $p c-W$ and rec$W$ represent polycrystalline tungsten and recrystallized tungsten, respectively.

The deuterium amounts measured by NRA and TPD are summarized in Fig. 5. Due to the limited information depth of NRA the D amount measured by NRA is in general lower than the D amount measured by TPD [7]. Both methods, NRA and TPD, show that the retained D amount reduces as a function of annealing temperature. The total retained $\mathrm{D}$ amounts in $\mathrm{W}$ films decrease from $1.65 \times 10^{21} \mathrm{D} / \mathrm{m}^{2}$ for the as-deposited film to $1.17 \times 10^{21} \mathrm{D} / \mathrm{m}^{2}$ after annealing at $1200 \mathrm{~K}$, and it further decreases to $0.95 \times 10^{21} \mathrm{D} / \mathrm{m}^{2}$ after annealing at $2000 \mathrm{~K}$. The fraction of $\mathrm{D}$ retained in the film relative to the total amount measured by TPD increases for these three samples from $71 \%$ over $81 \%$ to $88 \%$. The total D amount in the reference W bulk substrate $\left(0.47 \times 10^{21} \mathrm{D} / \mathrm{m}^{2}\right)$ is a factor of 2 lower than that of the $\mathrm{W}$ film annealed at $2000 \mathrm{~K}$. The D amount in the recrystallized bulk W sample was not measured by TPD. The D amount in this sample determined from NRA $\left(0.09 \times 10^{21}\right)$ corresponds to $20 \%$ of the reference $\mathrm{W}$ bulk substrate and to about $10 \%$ of the $\mathrm{W}$ film annealed at $2000 \mathrm{~K}$.

\subsection{Recrystallization of W film}

It was reported by Manhard et al. that D retention in bulk $\mathrm{W}$ annealed at $2000 \mathrm{~K}$ reduces by a factor of two compared with polycrystalline bulk $\mathrm{W}$ because most of the trap sites in bulk W, such as dislocations and grain boundaries, were significantly reduced due to the recrystallization process [9]. On the contrary, from Fig. 2 we can see that the D concentration in the top $500 \mathrm{~nm}$ of the $\mathrm{W}$ film after $2000 \mathrm{~K}$ annealing is four times higher compared with the as-deposited film. In Fig. 1 it is shown that annealing at $2000 \mathrm{~K}$ leads to the formation of clearly visible large cavities which accumulate at grain boundaries. Obviously, these cavities provide new trapping sites and it appears that the $\mathrm{D}$ retention in these cavities leads to a higher $\mathrm{D}$ retention than in the distributed bulk traps prior to annealing which are not visible by SEM.

Considering the fact that the recrystallization process strongly depends on the annealing temperature and holding time at this temperature two new experiments were carried out. In 
the one case, the $\mathrm{W}$ film deposited on polycrystalline tungsten was annealed at $2000 \mathrm{~K}$ in the protective gas atmosphere oven for 5 hours, i.e., for a 10-times longer holding time than before. In the other case, the $\mathrm{W}$ film deposited on polycrystalline tungsten was annealed at $2150 \mathrm{~K}$ for 6 minutes in the high vacuum oven. Due to technical limitations the sample cannot be kept longer than $6 \mathrm{~min}$ at the maximum temperature. The corresponding structural changes after these heat treatments were investigated by FIB cross sectioning in the SEM.
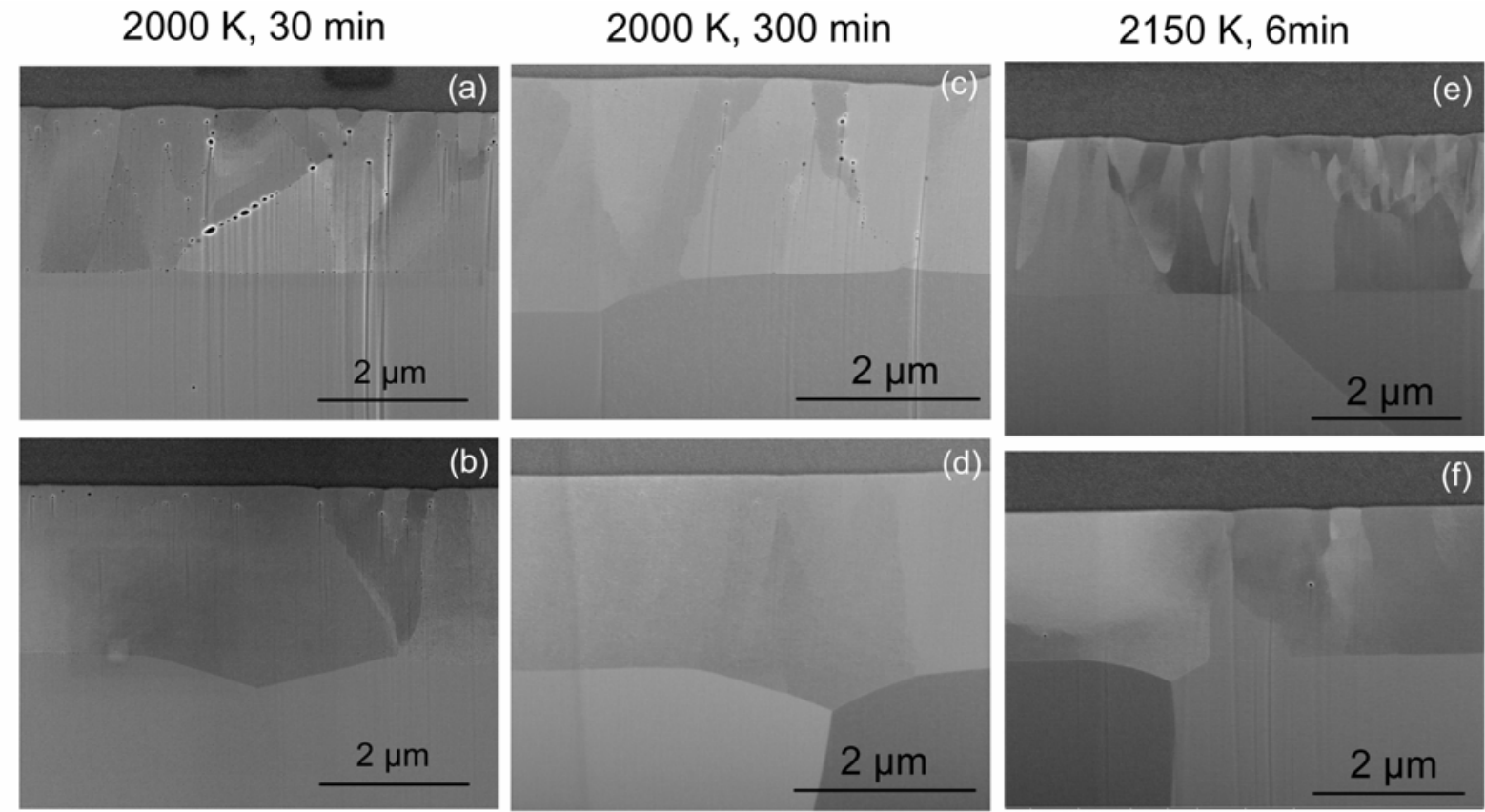

Fig. 6: Cross-section images of $W$ films after different heat treatments (two representative images are shown for each sample): (a) and (b) at $2000 \mathrm{~K}$ for 30 minutes, (c) and (d) at $2000 \mathrm{~K}$ for 300 minutes, (e) and (f) at $2150 \mathrm{~K}$ for 6 minutes. Note the magnifications of images (c) and (d) varies from others and for all images the angle between the electron beam and the crosssection plane is $38^{\circ}$ so that the vertical distances appear in the images shorter that they are in reality.

Fig. $6 \mathrm{a}, \mathrm{b}$ and $\mathrm{c}, \mathrm{d}$ shows cross-section images of $\mathrm{W}$ films after annealing at $2000 \mathrm{~K}$ for 30 and $300 \mathrm{~min}$, respectively. The $\mathrm{W}$ film after annealing at $2150 \mathrm{~K}$ for 6 minutes is shown in Figs. 6e and f. For each case, 2 representative images are shown. In the shown images we can clearly distinguish two different recrystallization zones: the $\mathrm{W}$ film and the $\mathrm{W}$ bulk. Due to comprehensive recrystallization and grain growth processes already after 30 min annealing at $2000 \mathrm{~K}$ only one or two grains are visible in the region of the initial bulk W substrates. The recrystallization behavior in the $\mathrm{W}$ films deposited on bulk $\mathrm{W}$ is very different. Even after 300 min at $2000 \mathrm{~K}$ or $6 \mathrm{~min}$ at $2150 \mathrm{~K}$ the initial interface between the $\mathrm{W}$ film and the bulk $\mathrm{W}$ is still visible and the film shows a distinctly different grain structure than the bulk. Within the $\mathrm{W}$ films we can also distinguish two different zones., One zone is characterized by grain with dimensions in the $\mu \mathrm{m}$ range (Figs. $6 \mathrm{~b}, 6 \mathrm{~d}$ and $6 \mathrm{e}$ ) and the other zone with significantly smaller grains with sizes down to the hundred nm range (Figs. 6a, 6c and 6f). Furthermore, it seems that the larger grains in the film have merged in many cases (Figs. 6b, 6d and 6e) with the top grain of the substrate while the smaller ones have not. Another noticeable difference between the samples after different heat treatment is the distribution of cavities. The density of cavities after the extended annealing at $2000 \mathrm{~K}$ (Figs. 6c, d) has significantly decreased compared with Figs. $6 \mathrm{a}$ and $6 \mathrm{~b}$ and after annealing at $2150 \mathrm{~K}$ hardly any cavities are visible. Apparently, magnetron-sputtered $\mathrm{W}$ films show a larger resilience against recrystallization than bulk W. One possible reason for that is that the initial grain size in the sputtered $\mathrm{W}$ film is smaller than bulk W. 


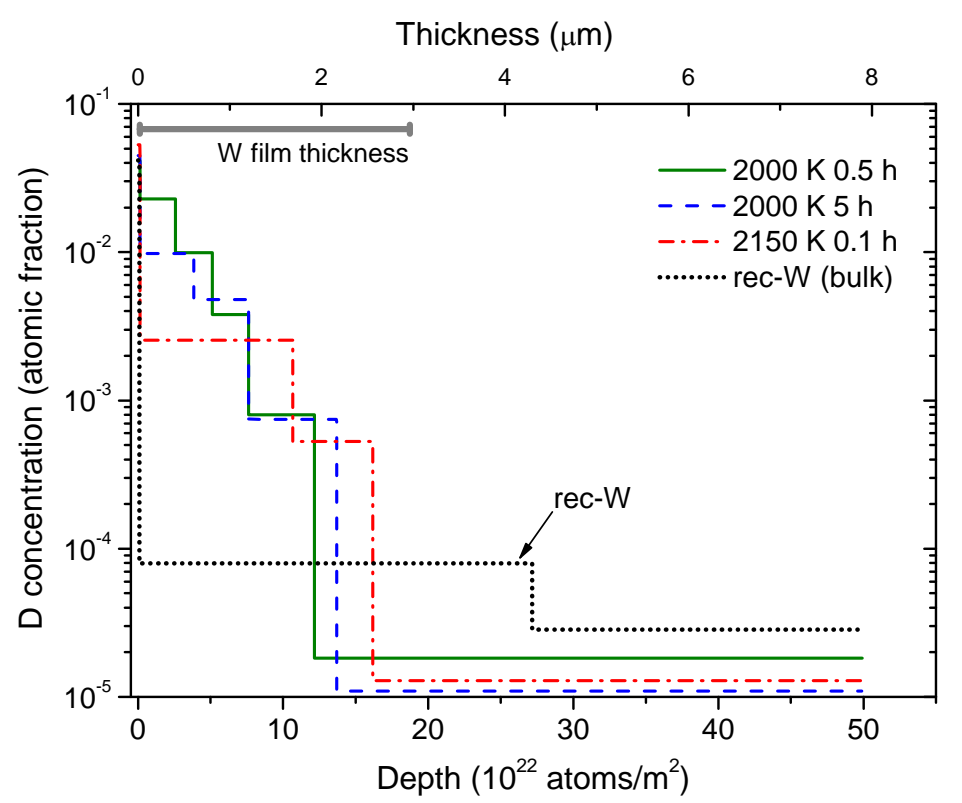

Fig. 7: Deuterium concentration depth profiles of $W$ films recrystallized at different parameters. All implantations were done at $370 \mathrm{~K}$ with $38 \mathrm{eV} / \mathrm{D}$ incident energy with a fluence of $6 \times 10^{24} \mathrm{D} / \mathrm{m}^{2}$. For comparison, $D$ depth profile of bulk $W$ annealed at $2000 \mathrm{~K}$ is also shown.

Fig. 7 shows the D depth profiles of recrystallized $\mathrm{W}$ film exposed to deuterium plasma. For comparison the D depth profile for recrystallized bulk W is also plotted. Apparently, both treatments, increased holding time at $2000 \mathrm{~K}$ and increased annealing temperature, lead to a decrease of the D concentration compared with the sample annealed at $2000 \mathrm{~K}$ for half hour. After the ten times longer holding time at $2000 \mathrm{~K}$ (300 minutes) the D concentration in the W film in first $500 \mathrm{~nm}$ region reduced by more than a factor of 2 to a value of $1 \times 10^{-2}$. The $\mathrm{D}$ concentration decreases further to $3 \times 10^{-3}$ after the $2150 \mathrm{~K}$ annealing. In both cases, D diffuses deeper into the film compared with the $\mathrm{W}$ film after annealing at $2000 \mathrm{~K}$ for $30 \mathrm{~min}$. From the cross-section images shown in Fig. 6 we can clearly see that the density of cavities has decreased after increasing holding time at $2000 \mathrm{~K}$ or after annealing at $2150 \mathrm{~K}$. Consequently, we attribute the observed decrease of the $\mathrm{D}$ concentration in the $\mathrm{W}$ films to the elimination of cavities due to annealing. Interestingly, with the decrease of the D concentration in the film D diffuses to the deeper regions. This observation is consistent with our earlier assumption that the presence of these cavities significantly slows down the penetration of D into films. Due to the much lower densities of these cavities in the additionally annealed films D can now diffuse into deeper regions. The total retained $\mathrm{D}$ amount determined from the NRA depth profiles reduced from $0.95 \times 10^{21} \mathrm{D} / \mathrm{m}^{2}$ to $0.85 \times 10^{21} \mathrm{D} / \mathrm{m}^{2}$ after $300 \mathrm{~min}$ at $2000 \mathrm{~K}$. It further reduced to $0.35 \times 10^{21} \mathrm{D} / \mathrm{m}^{2}$ after annealing at $2150 \mathrm{~K}$. However, even in the sample annealed at $2150 \mathrm{~K}$, which has the lowest observed $\mathrm{D}$ concentration in a recrystallized $\mathrm{W}$ film, the $\mathrm{D}$ concentration is still higher than in recrystallized bulk W.

\section{Summary}

Tungsten films deposited by magnetron-sputtering were used as a model system to study the influence of the film microstructure on the deuterium retention behavior. W films with different microstructures were produced by annealing the $\mathrm{W}$ film after deposition at different temperatures. Both TPD and NRA results prove that deuterium retention in the W films strongly depends on the microstructures of the films. The local deuterium concentrations vary by up to two orders of magnitude and the D retention in the films decreases as a function of annealing temperature. As-deposited W films show a typical columnar epitaxial growth with 
grain boundaries preferentially oriented perpendicular to the substrate surface. The D concentration in the as-deposited film corresponds to an atomic fraction about $6 \times 10^{-3}$. It is a factor of 3 higher than the D concentration in bulk tungsten. In this film blisters which are located close to the interface inside the W bulk material and not in the film or at the interface were observed in SEM surface images. These blisters cause an increase of the D concentration in the vicinity of the interface and lead to burst-like release events during TPD measurements. The released D amount in the $\mathrm{W}$ film annealed at $1200 \mathrm{~K}$ is about $30 \%$ lower than in the asdeposited samples and no blisters were observed in SEM surface images. No significant change of the films grain structure was observed in cross-section images after annealing at $1200 \mathrm{~K}$. Surprisingly, many grain boundaries are still present in the film after annealing at $2000 \mathrm{~K}$ while the average grain size in the $\mathrm{W}$ substrates is larger than the image size. In crosssection images bubble-like cavities were found. These cavities accumulated at the grain boundaries within the $\mathrm{W}$ film and the initial interface between the $\mathrm{W}$ film and $\mathrm{W}$ substrate. They provide abundant trap sites for D and result in a substantial increase of the local D concentration in the top $500 \mathrm{~nm}$. Surprisingly the local D concentration in this region is even higher than in the as-deposited films and the presence of these large cavities significantly slows down the penetration of D into this layer. While the chosen D fluence of $6 \times 10^{24} \mathrm{D} / \mathrm{m}^{2}$ was sufficient to saturate all other investigated samples even a 3 times larger fluence was not sufficient to completely fill this layer. Although a further increase of the holding time at $2000 \mathrm{~K}$ or an increase of the annealing temperature to $2150 \mathrm{~K}$ lead to the reduction of the cavity density and the retained $\mathrm{D}$ amount, the $\mathrm{D}$ concentration in the recrystallized $\mathrm{W}$ films cannot be reduced to a level as low as that of bulk W recrystallized at $2000 \mathrm{~K}$ for $30 \mathrm{~min}$. This indicates that magnetron sputtered $\mathrm{W}$ films have a higher resilience against recrystallization than bulk W.

\section{Acknowledgments}

The stay of P. Wang at Max-Planck Institute for Plasma Physics in Garching was funded through a bilateral agreement between Max-Planck Society and the Chinese Academy of Sciences which is gratefully acknowledged. This project has received funding from the EURATOM research and training program 2014-2018. 


\section{References}

[1] G. Federici, C. H. Skinner, J. N. Brooks, et al., Nucl. Fusion. 41 (2001) 1967.

[2] J. Roth, E. Tsitrone, A. Loarte, T. Loarer, et al., J. Nucl. Mater. 390-391 (2009) 1.

[3] R. A. Causey, J. Nucl. Mater. 300 (2002) 91.

[4] V. Kh. Alimov, J. Roth, Phys. Scr. T128 (2007) 6.

[5] O. V. Ogorodnikova, J. Roth, M. Mayer, J. Appl. Phys. 103 (2008) 034902.

[6] J. Roth, K. Schmid, Phys. Scr. T145 (2011) 014031.

[7] P. Wang, W. Jacob, L. Gao, T. Dürbeck, T. Schwarz-Selinger, Nucl. Instr. Meth. B 300 (2013) 54.

[8] P. Wang, W. Jacob, L. Gao, S. Elgeti, M. Balden, Phys. Scr. T159 (2014) 014046.

[9] A. Manhard, K. Schmid, M. Balden, W. Jacob, J. Nucl. Mater. 415 (2011) S632.

[10] A. Manhard, G. Matern, M. Balden, Practical Metallography 50 (2013) 5.

[11] B. Landkammer, A. von Keudell, W. Jacob, J. Nucl. Mater. 264 (1999) 48.

[12] T. Schwarz-Selinger, A. von Keudell, and W. Jacob, J. Appl. Phys. 86 (1999) 3988.

[13] A. Manhard, T. Schwarz-Selinger, W. Jacob, Plasma Sour. Sci. Technol. 20 (2011) 015010 .

Note: Unfortunately, the information given in the last paragraph of this article is not correct. The contribution of the molecular ions to the total ion flux for standard conditions is: $\mathrm{D}_{3}{ }^{+}=94 \%, \mathrm{D}_{2}{ }^{+}=3 \%$, and $\mathrm{D}^{+}=3 \%$. Correspondingly, the contributions to the total deuteron flux in form of ions are: $97 \%, 2 \%$, and $1 \%$ as expressed correctly in figures 5 and 6 in this reference.

[14] M. Mayer, „SIMNRA User’s Guide“, IPP Report Number: IPP 9/113, Max-PlanckInstitut für Plasmaphysik, Garching (1997).

[15] K. Schmid, U. von Toussaint, Nucl. Instr. Meth. B 281 (2012) 64.

[16] E. Salançon, T. Dürbeck, T. Schwarz-Selinger, F. Genoese, W. Jacob, J. Nucl. Mater. $376(2008) 160$.

[17] K. A. Moshkunov, K. Schmid, M. Mayer, V. A. Kurnaev, Yu. M. Gasparyan, J. Nucl. Mater. 404 (2010) 174.

[18] A. Manhard, U. von Toussaint, T. Dürbeck, K. Schmid, W. Jacob, Phys. Scr. T145 (2011) 014038 . 